Review Article

\title{
Time to Fix the Perceived Physical and Psychiatric Disanalogy
}

\section{Anum Haider}

AQ Khan Center, Institute of Behavioral Sciences, Karachi.

Corresponding Author: dranum.haider@gmail.com

\begin{abstract}
Health is a combination of good physical and mental well-being. Hence, it is important to cater both physical and psychiatric health elements equally. Current practice is mismatch of this ideology and both specialties are very much separately working. In this article, the association between these two health aspects along with underlying mechanisms, underpinning factors for such dissociation, appropriate recommendations and implications will be discussed.
\end{abstract}

\section{Keywords}

Physical, psychiatric, health, factors, mechanisms.

\section{Background}

According to the Health definition by WHO (World health Organization), the complete health is with both physical and mental (psychiatric) well-being of a person. If health compromises in either way it may hamper functioning or productivity of a person (Alonso, et al., 2011). However the research pool is not sufficient in relation to the demand of this subject. Nevertheless, to date studies have clearly mentioned the connection between physical and psychiatric disorders rather considering these as separate entities.

\section{The Linkage between Physical and Psychiatric Disorders}

They can present either as a comorbidity or as an associated condition.

\section{As a comorbidity}

A person with one kind of disorder is more susceptible to develop the other (Druss, 2011). Chronic physical conditions like cardiovascular diseases, Diabetes mellitus (D.M), Pulmonary diseases (Vogele, 2008), renal diseases(Moreira, 2008), neurological or dental diseases (Tomar, et al., 2011 \&
Kisley, et al., 2011), If not managed well, it can lead to psychiatric problems, most commonly depression, anxiety and suicide (Jones, et al., 2004). Almost half (58\%) of the population with medical problems develop psychiatric problems (Druss, 2011). Similarly chronic and severe psychiatric disorders (Depression, Bipolar disorder, Schizophrenia) can also produce physical problems such as cardiovascular disorders, D.M and Chronic pain (Hert, et al., 2009 \& Smith, 2011). More than half $(68 \%)$ of the population with mental disorder can develop comorbid medical problems (Druss, 2011).

\section{As an associated condition}

Psychiatric problems like depression are very much associated with physical problems that are chronic, multiple in number and poorly managed. (Gunn, et al., 2012). Physical problems may also manifest as psychiatric problems like SLE (systemic lupus erythromatosis) (Hajighaemi, 2016). Psychiatric problem may present as medical problems for instance, chronic pain syndromes. (Phillips, 2011). Richard et al. in his study re-explained that $46 \%$ of cases 
appear as medical disorders once reassessed in comparison with previously diagnosed psychiatric cases with full battery (physical, psychiatric, neurological exam and labs), (Nousin, et al., 2013)

\section{The Underpinning Mechanisms of}

\section{Linkage}

It is a well-established fact that inflammatory markers get increased when a person suffers from physical problems. The inflammatory markers are involved in almost all kind of physical illnesses in form of predisposing, triggering or maintaining factor. There are also similar evidences for psychiatric disorders in terms of potentiated proinflammatory mediators and inflammatory reactions and decreased immunity (Druss, 2011 \& Nousin, et al., 2013). Hence inflammation is a common underpinning factor that plays a bidirectional role in the manifestation of physical or psychiatric conditions (Druss, 2011). The proinflammatory markers and cytokines (CReactive Proteins, TNF-Tumor necrosis factor $\alpha$, gamma interferons, interleukin-6, interleukin-8) are responsible to execute inflammatory process and production of physical and psychiatric problems while on the other hand physical or psychiatric disorders may also turn on the inflammatory cascade (Druss, 2011 \& Leboyer, et al., 2012).

The common negative health behaviors in both kinds of disorders that are responsible to start the inflammatory cascade are poor diet, lack of exercise, alcohol, smoking or other psychoactive substance use (Druss, et al., 2011 \& Larsen, 2009). These negative health behaviors are commonly observed in patients with mental disorders and chronic medical problems. These patients also have shared genetic predisposition to develop metabolic disturbances and psychopathologies (Nousin, et al., 2013). Other than genetic basis, medications make the person susceptible to develop metabolic disturbances (obesity, impaired glucose tolerance, hypertension, and dyslipidemia) like antipsychotics as well anti-hypertensive or prescribed medications may cause psychological disturbances (Hert, et al., 2011). Metabolic disturbances may itself drift into various psychopathologies (schizophrenia, ADHD-Attention deficit hyperactivity disorder, ASD-Autism spectrum disorder), (Nousin, et al., 2013). Inflammatory process is good to handle the short term stress but when the stress becomes chronic or prolonged, it brings damaging health consequences by decreasing immunity and release of common pro-inflammatory mediators (cytokines, interleukins, tumor necrosis factors), (Sareen, 2007).

This results in devastating changes in neuronal structure and functions by alteration in synaptic proteins and intracellular signaling, loss of neurotropic support, inhibited neurogenesis, inhibited neuronal network connectivity, cytoskeletal destabilization and glutamate toxicity. These changes manifest as different neuropsychiatric and medical conditions (Duric, 2016). Broadly it disrupts the hypothalamic pituitary axis (HPA) and neuro-modulatory apparatus (like neurotransmitters). These are common risk factors that underpin this inflammatory cascade to bring various physical and psychiatric problems on the surface (Druss, 2011 \& Taylor, et al., 2012).

\section{Biological Factors}

- Genetics

- Leptin sensitivity

- Obesity

- Smoking or other psychoactive substance use.

- Poor nutrition 


\section{Psychological factors}

- Adverse life events

- Abuse/Neglect

- Chronic stressors

- Negative cognitive process and emotions

- Maladaptive personality traits

\section{Social factors}

- Poverty

- Poor social support

- Isolation

- Sedentary life style

- Poor neighborhood / household

Beside biological factors; the psychosocial factors (exposure to early life trauma, chronic stressors, and low socioeconomic status) have equal role in the causation of different medical and psychiatric disorders by disruption of HPA-axis (Druss, et al., 2011 \& Sareen, 2007). Hence there is a complex interplay of various neurobiological and psychosocial factors for the production of almost any kind of medical or psychiatric disorders (Druss, $2011 \&$ Taylor et al., 2012).

\section{Perception and Practice in the}

\section{Community}

Despite the strong association between these two kinds of disorders the comorbidity remains unattended or mislabeled as psychosomatic, functional or self-inducing at a significantly higher rate. It ultimately worsens the psychological stability, treatment adherence, quality of life and life expectancy (Phillips, et al., 2011 \& Gray, 2012). (Erwin, et al., 2011) in his study has found high rate of physical comorbidity in psychiatric disorders (43\%) and found both physician and psychiatrists to be least proficient in identification and management of such comorbidities and associated
Conditions both in primary care and mental health settings (Walker, 2011).

Factors behind This Perception

The ultimate question arises in mind," how such an important presentation can be overlooked by health professionals?" The research has proposed the possible answers:

-There are lack of expertise of psychiatrists to identify and treat medical conditions or they discount it due to lack of available resources. -The physicians at their end feel discomfort to treat medical conditions in psychiatric patients or take it for granted.

-There is lack of adequate communication and coordination between physician and psychiatrist.

\section{Consequences-Facts and Figures}

(Scoll, et al., 2009) have worked to measure the burden of disability due to physical (medical) and psychiatric problems. The disability due to psychiatric problems is high than physical problems and it carry remarkable synergistic effects on disability burden as a result of comorbidity with physical disorders. It increases the burden usually in two ways:

\section{Increasing the rate of comorbidity}

The properly unaddressed physical or psychiatric disorders result in high rate of comorbidity. In patients with severe mental illness there is high comorbidity with physical disorders like CVD, D.M, Pulmonary, dental. Similarly many chronic medical conditions may be worsened due to comorbid psychiatric conditions such as depression in diabetic patient decreases motivation for maintaining diet control, exercise and other stress relieving activities.

\section{Premature mortality}

Mortality gap is very higher (2-4 fold), around 15-20yrs earlier people with severe 
mental illness (i.e., schizophrenia) get die even in high income countries due to suicide and other unattended physical health conditions. Grossly on a broader view, theses unrecognized and unmanaged physical and psychiatric comorbidities affects person both at individual and community level. At individual level it impairs functioning, productivity and quality of life while on community level poses high economic burden (Druss \& Thornicroft, 2011; Gray, 2012 \& Lawrence, 2013).

\section{Conclusion}

The understanding of psychophysiological mechanisms made the clear relationship between physical and psychiatric disorders. The present health care system is not such integrated and coordinated to cater this realistic relationship. Clinical and policy implications are urgently needed to look into this matter.

\section{Implications and Recommendations}

\section{Clinical}

- Education and awareness of health professionals

Psychiatrists can play crucial role by expanding the clinical paradigm by inclusion of physical monitoring and evidence based prescription in daily practice along with education of patients regarding promotion of healthy life style and behaviors (Hert, et al., 2011). Nurses and primary care physicians are equally needed to be aware and educated about various common mental health problems in patients with medical illness (Hardy, et al., 2011 \& Bradshaw, 2012).

\section{- Improving communication}

Good level of communication is instrumental in any health setting especially in our setup where mostly mental health setting is separated from medical setting. There is crucial need to develop good communication bonding between the medical and mental health settings for appropriate referral and management (Druss, 2011).

- Monitoring of physical and mental health status and effect of treatment

The standardized monitoring tools and storage system must be formulated to record this monitoring and to appreciate and identify health disruptions at the earliest level (Carlier, 2012 \& Eldridge, 2011).

- Promotion of healthy health behavior

Healthy health behaviors like cessation of smoking and other psychoactive substances, exercise, healthy diet, proper sleep must be equally promulgated in all health settings (Happel, 2012).

- Involvement of significant others in patient's care

The significant others in patient's life can ensure good care act as a role model to emulate and maintain healthy behavers and habits in patients (Thoits, 2011).

\section{Policy}

The stake holders and government must do take it alarming and derive some policies accordingly, few are suggested below.

\section{- Expansion of health budget}

From the total health budget only $0.4 \%$ is allocated to mental health (Mental health atlas, 2014). Expansion of health budget is intensely needed for the provision of services adequately.

\section{- Integration of mental health in general health setup}

According to World health organization recommendation mental health facility must be as accessible as that of physical health. Depression declared to be the leading cause morbidity and mortality and found in almost $42 \%$ of the patients with physical diseases (WHO report 2017 
march). Hence it is essential to integrate both medical and psychiatric care.

\section{- Development of financial collaboration with international agencies}

In third world under developed countries like Pakistan international funding collaboration with the agencies targeting common area (i.e. substance use, suicide) can improve the care, evidence based working and research.

\section{- $\quad$ Building trained workforce}

There is scarcity of psychiatrists (mental health professionals) in relation to the given population size. Primary care physicians, nursing staff, lady health workers, social workers or voluntary community workers must have necessary training to work effectively in their circles to improve health globally. Behavioral sciences and psychiatry must be included in the curriculum of all medical students to develop adequate insight for career selection.

\section{- $\quad$ Prioritize preventive strategies}

Preventive strategies must be implemented both at primary and secondary level.

\section{Primary Level}

Global awareness and promotion of healthy life strategies like balanced diet, exercise, cessation of smoking and other psychoactive substances and acquiring good social circle.

\section{Secondary level}

Specific high risk groups are focused and targeted for specific health measures like chronic medically ill for screening and treatment of depression/ anxiety or other psychological issues.

This article may initiate further research under observational (such as, association of physical and psychiatric disorders in our population in both urban and rural setting) and interventional grounds (such as effect of education and monitoring on clinical outcome, liaison work).

\section{References}

- Alonso, J., Petukhova, M. V., Vilagut, G., Bromet, E. J., Hintov, H., \& Karam, E. G. (2013). Days totally out of role associated with common mental and physical disorders. The burdens of mental disorders: Global perspectives from the WHO World Mental Health surveys, 137-48.

- Bradshaw, T., \& Pedley, R. (2012). Evolving role of mental health nurses in the physical health care of people with serious mental health illness. International Journal of Mental Health Nursing, 21(3), 266-273.

- Carlier, I. V., Meuldijk, D., Van Vliet, I. M., Van Fenema, E., Van der Wee, N. J., \& Zitman, F. G. (2012). Routine outcome monitoring and feedback on physical or mental health status: evidence and theory. Journal of Evaluation in Clinical Practice, 18(1), 104-110.

- De Hert, M., Dekker, J. M., Wood, D., Kahl, K. G., Holt, R. I. G., \& Möller, H. J. (2009). Cardiovascular disease and diabetes in people with severe mental illness position statement from the European Psychiatric Association (EPA), supported by the European Association for the Study of Diabetes (EASD) and the European Society of Cardiology psychiatry, 24(6), 412-424.

- Duric, V., Clayton, S., Leong, M. L., \& Yuan, L. L. (2016). Comorbidity factors and brain mechanisms linking chronic stress and systemic illness. Neural plasticity, 2016.

- Eldridge, D., Dawber, N., \& Gray, R. (2011). A well-being support program 
for patients with severe mental illness: a service evaluation. BMC psychiatry, 11(1), 46.

- Goodell, S., Druss, B. G., Walker, E. R., \& MAT, M. (2011). Mental disorders and medical comorbidity. The Synthesis Project.

- Gray, R. (2012). Physical health and mental illness: a silent scandal. International Journal of Mental Health Nursing, 21(3), 191192.

- Gunn, J. M., Ayton, D. R., Densley, K., Pallant, J. F., Chondros, P., Herrman, H. E., \& Dowrick, C. F. (2012). The association between chronic illness, multimorbidity and depressive symptoms in an Australian primary care cohort. Social psychiatry and psychiatric epidemiology, 47(2), 175-184.

- Hajighaemi, F., Etemadifar, M., \& Bonakdar, Z. S. (2016). Neuropsychiatric manifestations in patients with systemic lupus erythematosus: A study from Iran. Advanced biomedical research, 5 .

- Hall, R. C., Gardner, E. R., Stickney, S. K., LeCann, A. F., \& Popkin, M. K. (1980). Physical illness manifesting as psychiatric disease: II. Analysis of a state hospital inpatient population. Archives of General Psychiatry, 37(9), 989-995.

- Happell, B., Davies, C., \& Scott, D. (2012). Health behaviour interventions to improve physical health in individuals diagnosed with a mental illness: A systematic review. International Journal of Mental Health Nursing, 21(3), 236247.

- Hardy, S., White, J., Deane, K., \& Gray, R. (2011). Educating healthcare professionals to act on the physical health needs of people with serious mental illness: a systematic search for evidence. Journal of Psychiatric and Mental Health Nursing, 18(8), 721727.

- Hert, M., Cohen, D. A. N., Bobes, J., Cetkovich-Bakmas, M. A. R. C. E. L. O., Leucht, S., Ndetei, D. M., \& Gautam, S. (2011). Physical illness in patients with severe mental disorders. II. Barriers to care, monitoring and treatment guidelines, plus recommendations at the system and individual level. World psychiatry, 10(2), 138-151.

- Hert, M., Correll, C. U., Bobes, J., Cetkovich-Bakmas, M. A. R. C. E. L. O., Cohen, D. A. N., Asai, I., ... \& Newcomer, J. W. (2011). Physical illness in patients with severe mental disorders. I. Prevalence, impact of medications and disparities in health care. World psychiatry, 10(1), 52-77.

- Hitchen, L. (2011). Psychiatrists neglect patients' physical health problems, says study. BMJ: British Medical Journal, 343.

- Jones DR, Macias C, Barreira PJ, et al. (2004). Prevalence, severity and cooccurrence of chronic physical health problems of persons with severe mental illness. Psychiatr Serv (11):1250-57.

- Kisley S, Quek L, Pais J, et al. (2011). Advanced dental disease in people with severe mental illness: systematic review and metanalysis. $\mathrm{Br} \quad \mathrm{J}$ Psychiatry (3):187-93

- Koryani EK. (1979). Morbidity and rate of undiagnosed physical illnesses in a psychiatric clinic population. Arch Gen Psychiatry (4):414-9.

- Larsen BA and Christenfeld NJS (2009). Cardiovascular disease and 
psychiatric comorbidity: The potential role of perseverative cognition. Cardiovascular Psychiatry Neurol. 791017:8 pages.

- Lawrence D, Hancock KJ, Kisley S. (2013). The gap in life expectancy from preventable physical illness in psychiatric patients in Western Australia: retrospective analysis of population based registers. BMJ 346:2539.

- Leboyer M, Soreca I, Scott J, et al. (2012). Can Bipolar disorder be viewed as a multisystem inflammatory disease? J Affect Disord (1):1-10.

- Mercer, S. W., Gunn, J., Bower, P., Wyke, S., \& Guthrie, B. (2012). Managing patients with mental and physical multimorbidity.

- Moreira, J. M., Matta, S. M. D., Kummer, A. M., Barbosa, I. G., Teixeira, A. L., \& Silva, A. C. S. (2014). Neuropsychiatric disorders and renal diseases: an update. Jornal Brasileiro de Nefrologia, 36(3), 396400.

- Nousen, E. K., Franco, J. G., \& Sullivan, E. L. (2013). Unraveling the mechanisms responsible for the comorbidity between metabolic syndrome and mental health disorders. Neuroendocrinology, 98(4), 254-266.

- Phillips, K., \& Clauw, D. J. (2011). Central pain mechanisms in chronic pain states-maybe it is all in their head. Best Practice \& Research Clinical Rheumatology, 25(2), 141154.

- Sareen, J., Cox, B. J., Stein, M. B., Afifi, T. O., Fleet, C., \& Asmundson, G. J. (2007). Physical and mental comorbidity, disability, and suicidal behavior associated with posttraumatic stress disorder in a large community sample. Psychosomatic medicine, 69(3), 242-248.

- Scott, D., \& Happell, B. (2011). The high prevalence of poor physical health and unhealthy lifestyle behaviours in individuals with severe mental illness. Issues in mental health nursing, 32(9), 589-597.

- Scott, K. M., Von Korff, M., Alonso, J., Angermeyer, M. C., Bromet, E., Fayyad, J., \& Haro, J. M. (2009). Mental-physical co-morbidity and its relationship with disability: results from the World Mental Health Surveys. Psychological medicine, 39(1), 33-43.

- Taylor, V. H., McIntyre, R. S., Remington, G., Levitan, R. D., Stonehocker, B., \& Sharma, A. M. (2012). Beyond pharmacotherapy: understanding the links between obesity and chronic mental illness. The Canadian Journal of Psychiatry, 57(1), 5-12.

- Thoits, P. A. (2011). Mechanisms linking social ties and support to physical and mental health. Journal of health and social behavior, 52(2), 145161.

- Thornicroft, G. (2011). Physical health disparities and mental illness: the scandal of premature mortality.

- Tomar B, Bhatia NK, Kumar P, et al. (2011). The Psychiatric and dental interrelationship. Delhi Psychiatry J (1).

- Vogele C, Leupoldt AV. (2008). Mental disorders in COPD. Respir Med.102:764-73.

- Zheng, Z., Zeng, Y., \& Wu, J. (2013). Increased neuroplasticity may protect against cardiovascular disease. International Journal of Neuroscience, 123(9), 599-608. 